\title{
Tackling smoking cessation systematically among inpatients with heart disease
}

\author{
Robert D. Reid PhD, Kerri-Anne Mullen PhD, Andrew L. Pipe MD
}

- Cite as: CMAJ 2018 March 26;190:E345-6. doi: 10.1503/cmaj.180125

See related article at www.cmaj.ca/lookup/doi/10.1503/cmaj.170377

igarette smoking is a causative factor in the development of coronary artery disease, contributing to atherosclerosis, thrombus formation and endothelial dysfunction; nicotine, carbon monoxide and oxidative gases contained in tobacco smoke play key roles in these processes. ${ }^{1}$ Smoking increases the risk of cardiovascular death roughly threefold; ${ }^{2}$ even minimal levels of smoking increase risk for major cardiovascular events and sudden cardiac death. ${ }^{3}$ Among smokers with coronary artery disease, meta-analyses of large observational trials show smoking cessation reduces risk of cardiovascular death by $36 \%$ over an average follow-up of five years. ${ }^{4}$ Smoking cessation is the most powerful modifier of cardiovascular risk. ${ }^{4}$ Yet patients admitted to Canadian hospitals often don't receive treatment for their tobacco use despite the fact that effective treatments are available.

In linked research, Windle and colleagues report long-term findings of a double-blind, placebo-controlled trial of varenicline for smoking cessation after hospital admission for acute coronary syndrome (myocardial infarction or unstable angina). ${ }^{5}$ Twenty-four week outcomes from the Evaluation of Varenicline in Smoking Cessation for Patients Post-Acute Coronary Syndrome (EVITA) trial - the first clinical trial to report effects of varenicline in smokers with acute coronary artery disease who received their first dose of medication within two days of hospital admission - were previously published. ${ }^{6}$ The linked article reports outcomes at 52 weeks. Patients receiving varenicline were more likely to be smoke-free one year after hospital admission for acute coronary syndrome compared with placebo (39.9\% v. $29.1 \%$, difference $10.7 \%$, $95 \%$ confidence interval [CI] $0.01 \%$ to $21.4 \%$ ). Although the study was not powered to allow conclusions about the safety of varenicline, this rigorous, wellconducted study showed that varenicline and placebo groups had similar rates of serious adverse events $(24.5 \%$ v. $21.9 \%$, risk difference $2.7 \%, 95 \% \mathrm{Cl}-7.3 \%$ to $12.6 \%$ ) and major adverse cardiovascular events $(8.6 \%$ v. $9.3 \%$, risk difference $-0.7 \%, 95 \% \mathrm{Cl}$ $-7.8 \%$ to $6.5 \%)$. The study design allows us to conclude that adding varenicline to assist with smoking cessation, at the time of presentation with acute coronary syndrome, significantly increases cessation rates over the longer term.

\section{KEY POINTS}

- Quitting smoking should be a priority for patients presenting to hospital with acute coronary syndrome to reduce future cardiovascular morbidity and mortality, and to increase quality of life.

- The best way for smokers with acute coronary syndrome to quit smoking is with a combination of counselling and medication.

- Smoking cessation interventions should be initiated in the hospital and integrated into postdischarge support.

- Varenicline, a partial agonist and partial antagonist of a nicotinic receptor, is safe and efficacious in smokers presenting with acute coronary syndrome and can be integrated into treatment protocols for patients with these conditions.

Even with treatment, most participants in this trial had returned to smoking by 52-week follow-up. Tobacco addiction is a chronic, relapsing condition, and long-term abstinence is difficult to achieve for many smokers. ${ }^{7}$ Nicotine inhaled in tobacco smoke binds to specific receptors in the brain, triggering the release of neurotransmitters (principally dopamine) and creating a pleasant, rewarding sensation. Nicotine dependence develops quickly, and smokers experience symptoms of nicotine withdrawal (e.g., cravings, difficulty concentrating, irritability and depression) when they try to abstain from smoking; many smokers return to smoking as a consequence. Additionally, smoking becomes a highly conditioned behaviour as smokers associate the rewarding effects of smoking with daily routines, social situations, and positive and negative mood states. These settings, situations and circumstances can also trigger relapse during attempts to quit smoking. Successful treatments, therefore, combine pharmacotherapy with behavioural support. ${ }^{8}$

Previous research has confirmed the efficacy of varenicline in patients with stable cardiovascular disease. ${ }^{9}$ In a randomized controlled trial (RCT) with 714 smokers, patients receiving varenicline achieved a continuous abstinence rate of $19.2 \%$ for weeks 9-52 compared with $7.2 \%$ in the placebo group (odds ratio 3.14 , $95 \% \mathrm{Cl} 1.93$ to 5.11 ). ${ }^{9}$ Network meta-analyses confirm varenicline as the most efficacious single medication, ${ }^{10}$ although combina- 
tion treatments employing slow- and fast-acting nicotine replacement therapies can have similar efficacy. ${ }^{8}$

Safety data from the high-risk cardiovascular patients in the study by Windle and colleagues are reassuring and consistent with aggregate data from existing RCTs. Although RCTs are the best sources of evidence of safety, studies to date have not been adequately powered to allow conclusions about the cardiovascular safety of varenicline. Clinicians and decision-makers have therefore relied on meta-analyses to draw conclusions about varenicline's safety. The largest meta-analysis of the cardiovascular safety of varenicline to date, which was published recently, included data from 38 studies and 12706 smokers, including those with and without pre-existing cardiovascular disease. ${ }^{11}$ The occurrence of the combination of cardiovascular serious adverse events and mortality was $0.79 \%$ in the varenicline group versus $0.78 \%$ in the placebo group. Results were similar between those with and without pre-existing cardiovascular disease. ${ }^{11}$ It is clear that the benefits of improved cardiovascular health from pharmacotherapy-assisted smoking cessation far outweigh the putative risk of medication-induced cardiovascular harm.

Hospital admission for acute coronary syndrome (as well as other smoking-related conditions) often coincides with a spike in motivation and desire to quit smoking. Further, hospitals in Canada are smoke-free; the period of hospital admission offers an interval of enforced abstinence. Thus, it is opportune to ensure that smoking cessation interventions are integrated into routine hospital practices. These results and others underscore the pressing need to introduce systematic approaches to the delivery of smoking cessation interventions in hospitals across Canada. The Ottawa Model for Smoking Cessation (OMSC) has been implemented in many settings in Canada. ${ }^{12}$ The OMSC is an organizational and practice-level intervention that ensures that systematic processes to identify, treat and provide follow-up to hospitaladmitted smokers are in place. Pragmatic research examining the effect of implementing systematic, standard approaches like the
OMSC shows that implementation leads to relatively greater longterm quitting success $(+61 \%)$ and reduced hospital readmission $(-26 \%)$ and mortality $(-48 \%)$ among smokers. ${ }^{12}$

Given the powerful effect of smoking cessation on subsequent cardiovascular morbidity and mortality, smoking cessation interventions including counselling and medications, initiated in the hospital and integrated into postdischarge support, should be standard practice for patients with acute coronary syndrome receiving treatment at hospitals in Canada. Anything less reflects substandard care.

\section{References}

1. Rigotti NA, Clair C. Managing tobacco use: the neglected cardiovascular disease risk factor. Eur Heart J 2013;34:3259-67.

2. Yusuf S, Hawken S, Ounpuu S, et al. Effect of potentially modifiable risk factors associated with myocardial infarction in 52 countries (the INTERHEART study): case-control study. Lancet 2004;364:937-52.

3. Hackshaw A, Morris JK, Boniface S, et al. Low cigarette consumption and risk of coronary heart disease and stroke: meta-analysis of 141 cohort studies in 55 study reports. BMJ 2018;360:j5855.

4. Critchley JA, Capewell S. Mortality risk reduction associated with smoking cessation in patients with coronary heart disease: a systematic review. JAMA 2003;290:86-97.

5. Windle SB, Dehghani P, Roy N, et al. Smoking abstinence 1 year after acute coronary syndrome: follow-up from a randomized controlled trial of varenicline in patients admitted to hospital. CMAJ 2018;190:E347-54.

6. Eisenberg MJ, Windle SB, Roy N, et al. Varenicline for smoking cessation in hospitalized patients with acute coronary syndrome. Circulation 2016;133:21-30.

7. Benowitz NL. Nicotine addiction. N Engl J Med 2010;362:2295-303.

8. Reid RD, Pritchard G, Walker K, et al. Managing smoking cessation. CMAJ 2016;188:E484-92.

9. Rigotti NA, Pipe AL, Benowitz NL, et al. Efficacy and safety of varenicline for smoking cessation in patients with cardiovascular disease: a randomized trial. Circulation 2010;121:221-9.

10. Cahill K, Stevens S, Perera R, et al. Pharmacological interventions for smoking cessation: an overview and network meta-analysis. Cochrane Database Syst Rev 2013(5)CD009329.

11. Sterling LH, Windle SB, Filion KB, et al. Varenicline and adverse cardiovascular events: a systematic review and meta-analysis of randomized controlled trials. $J$ Am Heart Assoc 2016;5: pii: e002849.

12. Mullen KA, Manuel DG, Hawken SJ, et al. Effectiveness of a hospital-initiated smoking cessation programme: 2-year health and healthcare outcomes. Tob Control 2017;26:293-9.
Competing interests: Robert Reid has received speaking fees and a research grant from Pfizer, the maker of varenicline, and has received speaking fees from Johnson \& Johnson, which manufactures smokingcessation therapies. Kerri-Anne Mullen has received speaking fees from Pfizer. Andrew Pipe has received funding, and served as a consultant to, Pfizer and Johnson \& Johnson. All of the authors are inventors of the Ottawa Model for Smoking Cessation, a registered trademark of the University of Ottawa Heart Institute.

This article was solicited and has not been peer reviewed.
Affiliations: Divisions of Prevention and Rehabilitation and of Cardiology, University of Ottawa Heart Institute, and Faculty of Medicine, University of Ottawa, Ottawa, Ont.

Contributors: All of the authors made substantial contributions to the conception of the article. Robert Reid drafted the article, which Kerri-Anne Mullen and Andrew Pipe revised. All of the authors provided final approval of the version to be published and agreed to be accountable for all aspects of the work.

Correspondence to: Robert Reid, breid@ottawaheart.ca 